OPEN ACCESS

Edited by:

Yang Zhang,

University of Houston, United States

Reviewed by:

Ningjun Li,

Virginia Commonwealth University,

United States

Krishna M. Boini,

University of Houston, United States

Fangxiong Shi,

Nanjing Agricultural University, China

*Correspondence:

Zhenghong Zhang

zhangzh@finu.edu.cn

Specialty section:

This article was submitted to

Molecular Medicine,

a section of the journal

Frontiers in Cell and Developmental

Biology

Received: 22 August 2019 Accepted: 16 October 2019 Published: 30 October 2019

Citation:

Zhang Z, Wang F and Zhang Y

(2019) Expression and Contribution

of NLRP3 Inflammasome During

the Follicular Development Induced by

PMSG. Front. Cell Dev. Biol. 7:256.

doi: 10.3389/fcell.2019.00256

\section{Expression and Contribution of NLRP3 Inflammasome During the Follicular Development Induced by PMSG}

\author{
Zhenghong Zhang*, Fan Wang and Yan Zhang \\ Provincial Key Laboratory for Developmental Biology and Neurosciences, College of Life Sciences, Fujian Normal University, \\ Fuzhou, China
}

Follicular development and following ovulation induced by luteinizing hormone (LH) surge are critical for ovarian functions, but the molecular mechanism regulating ovarian ovulation attracts more attention and remains mainly unknown. Recent researches on the nucleotide leukin rich polypeptide 3 (NLRP3) inflammasome shred light on it. Given pregnant mare serum gonadotropin (PMSG) can not only trigger the follicular development, but also induce the following ovulation, the present study therefore examined that expression and localization of NLRP3 inflammasome through immunohistochemistry and Western blotting during the follicular development induced by PMSG. The results showed expressions of NLRP3 and the adaptor protein apoptosis-associated speck-like protein (ASC) significantly increased in the outside of intrafollicular fluid, further analysis found that caspase-1 was activated and IL$1 \beta$ production was also upregulated after $52 \mathrm{~h}$-treatment of PMSG. Furthermore, a significant increase of ovulation-related genes, hypoxia inducible factor (HIF)- $1 \alpha$ and endothelin (ET)-1, was found after 52 h-treatment of PMSG. To our knowledge, it is the first time to clearly indicated the activation of NLRP3 inflammasome may contribute to the ovulation of PMSG-treated ovaries, which will help to further clarify the ovulatory mechanism in mammals.

Keywords: NLRP3 inflammasome, pregnant mare serum gonadotropin, follicular development, ovulation, mouse

\section{INTRODUCTION}

In mammals, the ovary is the reproductive organs of females, and its main function is to produce oocytes and steroids. At present, it's believed that follicular development is triggered by folliclestimulating hormone (FSH) and the following ovulation is induced by luteinizing hormone (LH) surge (Komatsu and Masubuchi, 2016; Jiang et al., 2018; Qi et al., 2018; Wei et al., 2018). Pregnant mare serum gonadotropin (PMSG) can not only trigger the follicular development mainly similar to FSH, but also induce the following ovulation like $\mathrm{LH}$, which was widely used in the field of reproductive investigation (Ma et al., 1997; Tarín et al., 2002; Nie et al., 2018; Kim et al., 2019). It is worth noting that Espey put forward the hypothesis of ovulation as an inflammatory reaction (Espey, 1980), but the detailed mechanism regulating ovarian ovulation still remains unknown. Until recently, the researches on the nucleotide leukin rich polypeptide 3 (NLRP3) inflammasome shred light on it (Ahechu et al., 2018; Groslambert and Py, 2018; de Alba, 2019; Komada and Muruve, 2019; Takahashi, 2019; Yang et al., 2019). 
The inflammasome is the cellular machinery responsible for activation of inflammatory reaction (Mariathasan et al., 2004; Zhang et al., 2012; Li et al., 2014), which includes four types, like NLRP1, NLRP3, IPAF, and AIM2 inflammasome (Boini et al., 2014; Abais et al., 2015; Chen et al., 2016). NLRP3 inflammasome is a proteolytic complex of NLRP3, ASC, and caspase-1, which is well characterized (Boini et al., 2014; Abais et al., 2015; Chen et al., 2015; Zhang et al., 2015; Chen et al., 2016; Wang et al., 2016). Upon activation, NLRP3 binds to the ASC, which in turn recruits pro-caspase-1 to form an integrated inflammasome complex (Zhang et al., 2012; Boini et al., 2014; Li et al., 2014; Abais et al., 2015; Chen et al., 2015, 2016; Zhang et al., 2015; Wang et al., 2016), and subsequently cleaves pro-caspase-1 into activated caspase-1, leading to the production of matured IL-1 $\beta$ (Zhang et al., 2012; Boini et al., 2014; Li et al., 2014; Abais et al., 2015; Chen et al., 2015; Zhang et al., 2015; Chen et al., 2016; Wang et al., 2016), which is an important inflammatory cytokine (Zhang et al., 2012; Boini et al., 2014; Li et al., 2014; Abais et al., 2015; Chen et al., 2015; Zhang et al., 2015; Chen et al., 2016; Wang et al., 2016). However, the expression and contribution of NLRP3 Inflammasome during follicular development and ovarian ovulation still unknown.

Given the parallels of ovulation with inflammatory processes and the correlation of non-steroidal anti-inflammatory drug use with reversible infertility (Akil et al., 1996; Mendonça et al., 2000; Duffy et al., 2019), the present study therefore utilized animal model to examine that expression and localization of NLRP3 inflammasome through immunohistochemistry and Western blotting during the follicular development induced by PMSG for help to further clarify the ovulatory mechanism in mammals.

\section{MATERIALS AND METHODS}

\section{Experimental Design}

Immature female C57BL/6 mice (21-day old) were purchased from Wushi Experimental Animal Supply Co. Ltd. (Fuzhou, China) and maintained in the Laboratory Animal Center of Fujian Normal University under a 14-h light/10-h dark schedule with continuous supply of chow and water. The follicular development was induced in the mice treated with $10 \mathrm{IU}$ PMSG (i.p., Sigma-Aldrich, St. Louis, MO, United States) for 0,24 , and $52 \mathrm{~h}$. The left ovary of each animal was fixed in $4 \%$ paraformaldehyde for immunohistochemistry, and the right one was snap-frozen for the examination of gene and protein expression levels. The experiment was repeated two times.

\section{Immunohistochemistry}

After fixation, 5- $\mu \mathrm{m}$ sections were processed for immunohistochemical analysis with anti-NLRP3 antibody (1:500, Abcam, Cambridge, MA, United States), anti-ASC antibody (1:500, Abcam, Cambridge, MA, United States) and anti-IL-1 $\beta$ antibody (1:500, Abcam, Cambridge, MA, United States). The negative control used serum (Boster Biological Technology, Wuhan, China) instead of primary antibody and the immunoreactivity was visualized by the Elite ABC kit (BioGenex, San Ramon, CA, United States). Three
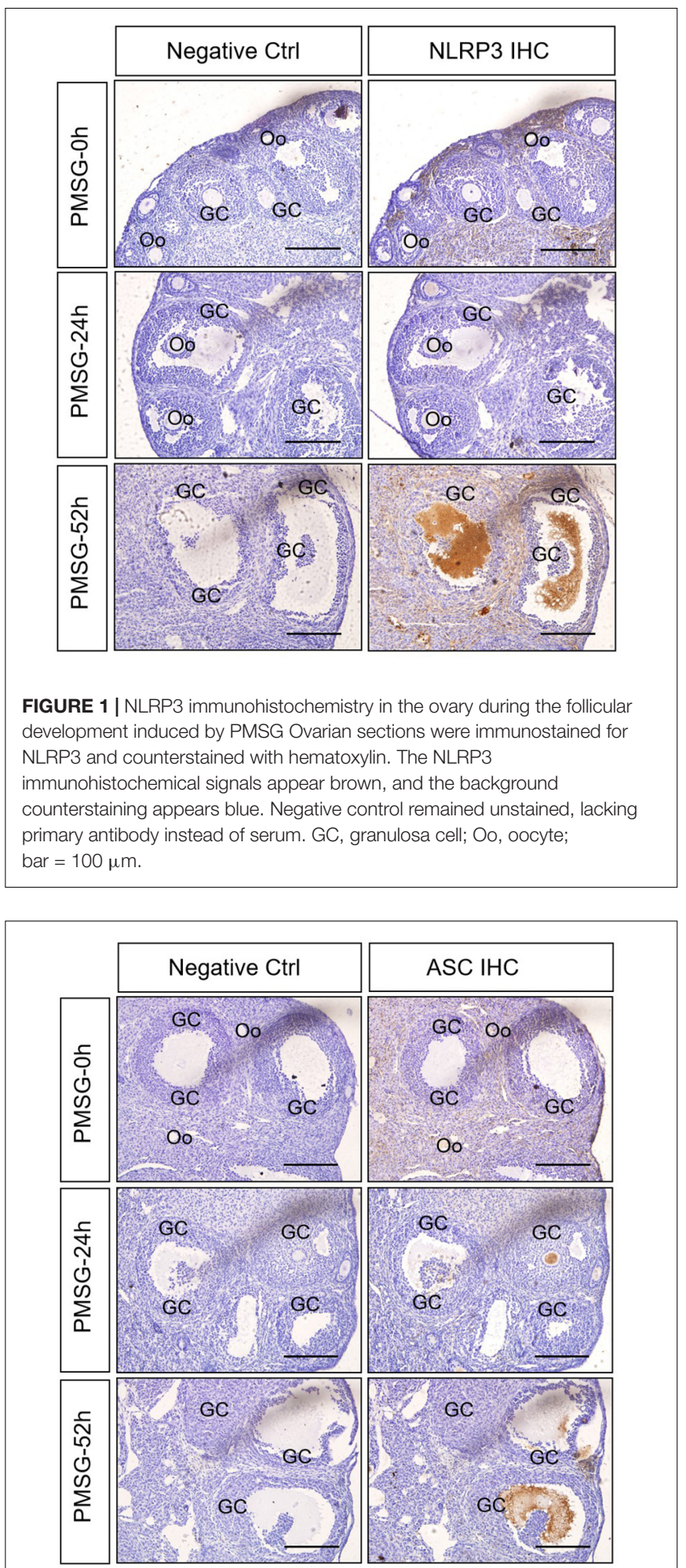

FIGURE 2 | ASC immunohistochemistry in the ovary during the follicular development induced by PMSG Ovarian sections were immunostained for ASC and counterstained with hematoxylin. The ASC immunohistochemical signals appear brown, and the background counterstaining appears blue. Negative control remained unstained, lacking primary antibody instead of serum. GC, granulosa cell; Oo, oocyte; bar = $100 \mu \mathrm{m}$. 
independent observers were asked to assess the intensity of immunostaining (Shi et al., 2004; Kim et al., 2005) and the evaluation of relative staining levels was repeated at least four times (Zhang W. et al., 2011).

\section{Western Blot Analysis}

Cytoplasmic protein extracts were prepared with the Protein Extraction Kit (Beyotime Biotechnology, Shanghai, China) according to the manufacturer's instructions, and the concentrations were determined by a BCA assay kit (Beyotime Biotechnology, Shanghai, China). These proteins were used for

TABLE 1 | Relative abundances of NLRP3 in the ovary during follicular development induced by PMSG.

\begin{tabular}{lccc}
\hline & \multicolumn{3}{c}{ Staining intensity } \\
\cline { 2 - 4 } Follicular development & PMSG-0 h & PMSG-24 h & PMSG-52 h \\
\hline Oocyte & & NA & NA \\
Primordial & - & - & + \\
Primary & - & + & + \\
Secondary & NA & NA & + \\
Tertiary & & & \\
Granulosa cells & - & NA & NA \\
Primordial & - & - & - \\
Primary & - & - & - \\
Secondary & NA & NA & - \\
Tertiary & & & \\
Theca cells & + & + & + \\
Secondary & NA & NA & + \\
Tertiary & + & + & ++ \\
Endothelial cells & - & - & +++ \\
Follicular fluid & &
\end{tabular}

-, no staining detected; +, weak; ++, moderate; +++, strong; NA, not available.

TABLE 2 | Relative abundances of ASC in the ovary during follicular development induced by PMSG.

\begin{tabular}{lccc}
\hline Follicular development & \multicolumn{3}{c}{ Staining intensity } \\
\cline { 2 - 4 } & PMSG-0 h & PMSG-24 h & PMSG-52 h \\
\hline Oocyte & & NA & NA \\
Primordial & - & - & + \\
Primary & + & ++ & ++ \\
Secondary & NA & NA & ++ \\
Tertiary & & & \\
Granulosa cells & - & NA & NA \\
Primordial & - & - & - \\
Primary & - & - & - \\
Secondary & NA & NA & - \\
Tertiary & & & - \\
Theca cells & - & - & - \\
Secondary & NA & NA & - \\
Tertiary & - & - & +++ \\
Endothelial cells & - & - & - \\
Follicular fluid & &
\end{tabular}

-, no staining detected; +, weak; ++, moderate; +++, strong; NA, not available. the following examination of expression levels and activities. The expression levels of different proteins were analyzed by Western blotting as described previously (Zhang Z. et al., 2011). NLRP3 antibody (1:1000, Abcam, Cambridge, MA, United States), ASC antibody 1:1000, Abcam, Cambridge, MA, United States), Pro-caspase-1 antibody (1:1000, Abcam, Cambridge, MA, United States), cleaved-caspase-1 antibody (1:1000, Abcam, Cambridge, MA, United States), IL-1 $\beta$

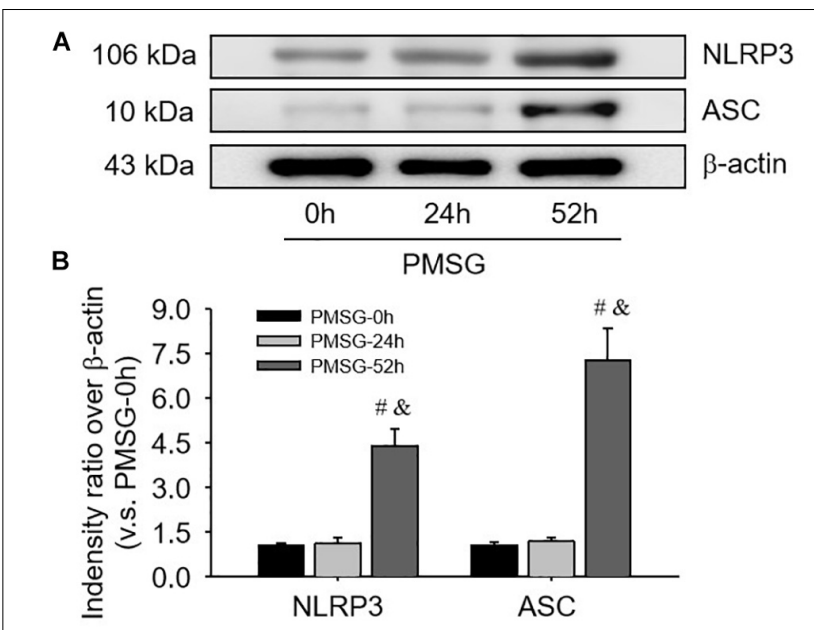

FIGURE 3 | NLRP3 and ASC protein expressions in the ovary during the follicular development induced by PMSG. (A) Representative ECL gel images of Western blot analyses depicting the NLRP3 and ASC protein levels. (B) Summarized intensities of NLRP3 and ASC blots normalized to the control. Each value represents the mean $\pm \mathrm{SE}$. One-way analysis of variance (ANOVA) was used to analyze the data, followed by a Tukey's multiple range test. $n=6$. ${ }^{\#} P<0.05$, vs. PMSH-0 h; $\& P<0.05$, vs. PMSH-24 h.

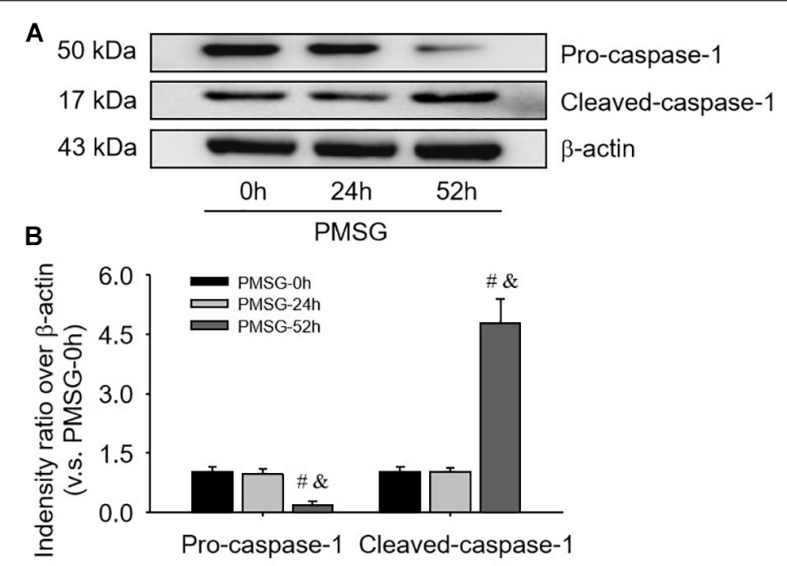

FIGURE 4 | Pro-caspase-1 and cleaved-caspase-1 protein expressions in the ovary during the follicular development induced by PMSG. (A) Representative ECL gel images of Western blot analyses depicting the pro-caspase-1 and cleaved-caspase-1 protein levels. (B) Summarized intensities of pro-caspase-1 and cleaved-caspase-1 blots normalized to the control. Each value represents the mean \pm SE. One-way analysis of variance (ANOVA) was used to analyze the data, followed by a Tukey's multiple range test. $n=6$. ${ }^{\#} P<0.05$, vs. PMSH-0 h; \& $P<0.05$, vs. PMSH-24 h. 


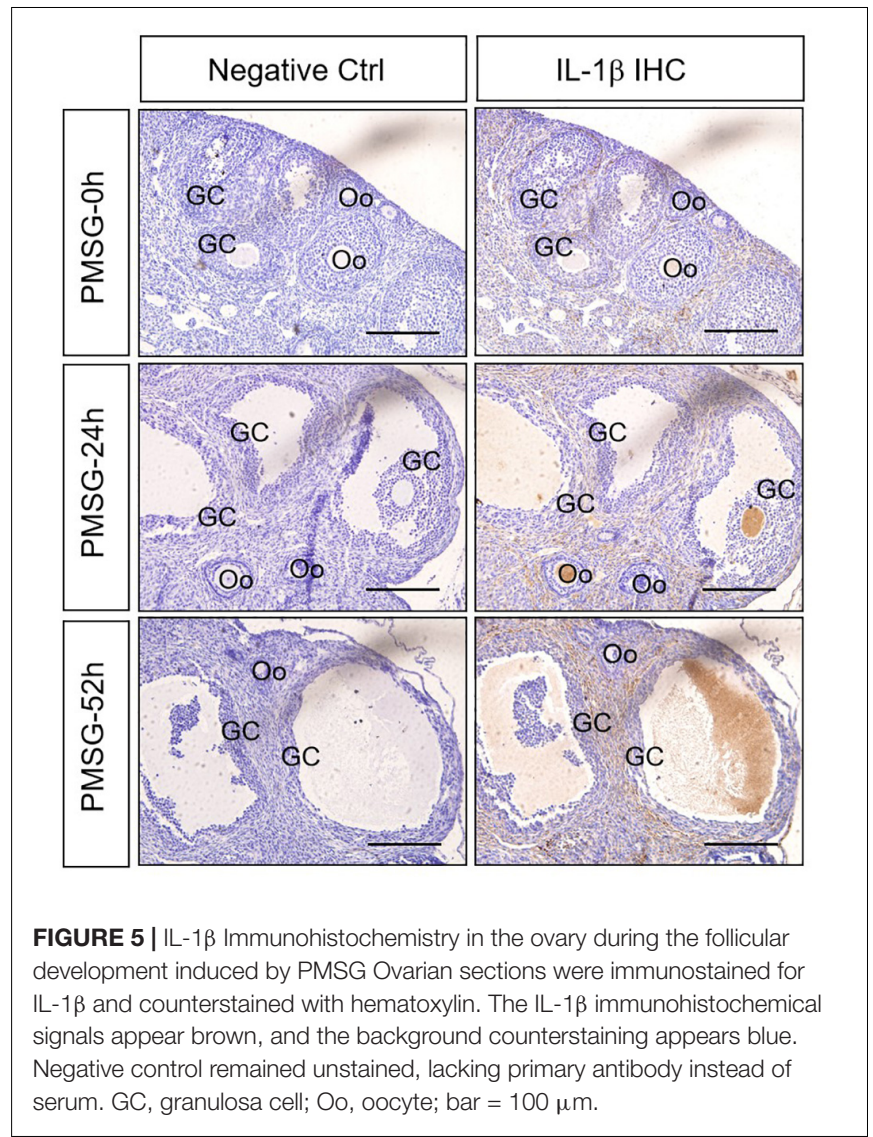

TABLE 3 | Relative abundances of IL-1 $1 \beta$ in the ovary during follicular development induced by PMSG.

\begin{tabular}{lccc}
\hline Follicular development & \multicolumn{3}{c}{ Staining intensity } \\
\cline { 2 - 4 } & PMSG-0 h & PMSG-24 h & PMSG-52 h \\
\hline Oocyte & - & NA & NA \\
Primordial & + & + & + \\
Primary & ++ & +++ & +++ \\
Secondary & NA & NA & +++ \\
Tertiary & & & \\
Granulosa cells & - & NA & NA \\
Primordial & - & - & - \\
Primary & - & - & - \\
Secondary & NA & NA & - \\
Tertiary & & & \\
Theca cells & + & + & + \\
Secondary & NA & NA & ++ \\
Tertiary & - & - & - \\
Endothelial cells & - & - & +++ \\
Follicular fluid & & &
\end{tabular}

-, no staining detected; +, weak; ++, moderate; +++, strong; NA, not available.

antibody (1:500, Abcam, Cambridge, MA, United States) and $\beta$-actin antibody (1:5000, Novus Biologicals, Littleton, CO, United States) were used.
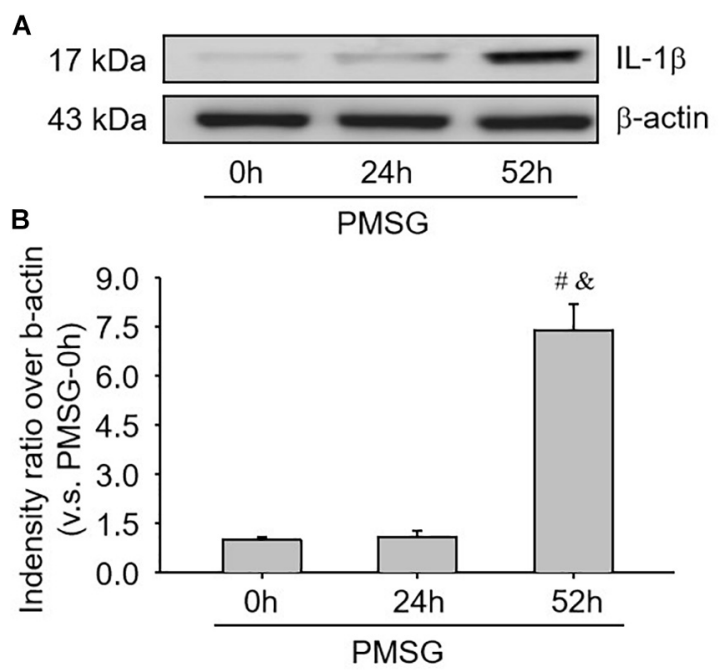

FIGURE 6 | IL-1 $\beta$ protein expressions in the ovary during the follicular development induced by PMSG. (A) Representative ECL gel images of Western blot analyses depicting the IL-1 $1 \beta$ protein levels. (B) Summarized intensities of IL-1 $\beta$ blots normalized to the control. Each value represents the mean \pm SE. One-way analysis of variance (ANOVA) was used to analyze the data, followed by a Tukey's multiple range test. $n=6$. ${ }^{\#} P<0.05$, vs. $\mathrm{PMSH}-\mathrm{O}$ h; ${ }^{\&} \mathrm{P}<0.05$, vs. $\mathrm{PMSH}-24 \mathrm{~h}$.

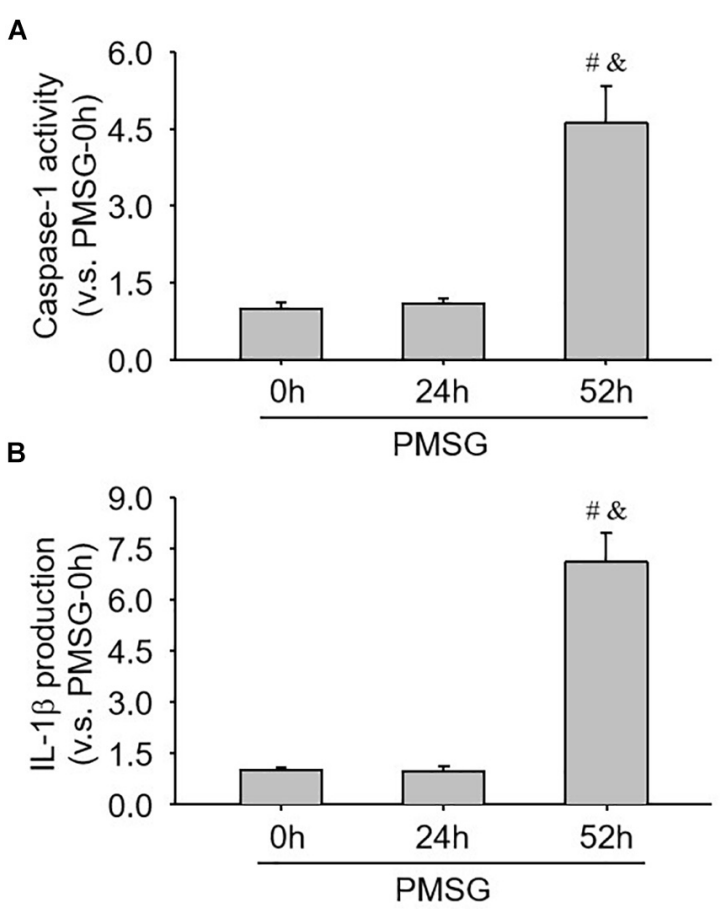

FIGURE 7 | Examination of caspase- 1 activity and IL- $1 \beta$ production in the ovary during the follicular development induced by PMSG. (A) The data for caspase-1 activity in the ovaries from each group normalized to the control (PMSG-0 h). (B) The data for IL-1 $\beta$ production in the ovaries from each group normalized to the control (PMSG-O h). Each value represents the mean $\pm \mathrm{SE}$. One-way analysis of variance (ANOVA) was used to analyze the data, followed by a Tukey's multiple range test. $n=6 .{ }^{\#} P<0.05$, vs. PMSH-0 h; ${ }^{\&} P<0.05$, vs. $\mathrm{PMSH}-24 \mathrm{~h}$. 


\section{Real Time PCR Analysis}

Total RNA was extracted using TRIzol (Life Technologies, Carlsbad, CA, United States) and then reverse-transcribed (cDNA Synthesis Kit, Bio-Rad, Hercules, CA, United States). The reverse-transcribed products were amplified using a TaqMan Gene Expression Assay kit (Applied Biosystems, Foster City, CA, United States), including TaqMan Universal PCR Master Mix, hypoxia-inducible factor (HIF)-1 $\alpha$ primer (Hs00936372_m1) and endothelin (ET)-1 primer (Mm00438656_m1). The levels of 18S ribosomal RNA (Rn03928990_g1) was used as an endogenous control. The relative gene expressions were calculated with the $\Delta \Delta \mathrm{Ct}$ method. Relative mRNA levels were expressed as $2^{-\Delta \Delta \mathrm{Ct}}$ values.

\section{Caspase-1 Activity and IL-1 $\beta$ Production Assay}

The assay of caspase-1 activity was performed by Caspase-1 Colorimetric Assay Kit (Biovision, Milpitas, CA, United States) and the level of IL-1 $\beta$ production was measured by IL-1 $\beta$ ELISA Kit (Bender Medsystems, Burlingame, CA, United States) according to the protocol described by the manufacturer. These data were expressed as the fold change compared with the control.

\section{Statistics}

Data are presented as mean \pm SE. The significance of differences in mean values within multiple groups was evaluated using a one-way analysis of variance (ANOVA), followed by a Tukey's multiple range test. $P<0.05$ was considered statistically significant.

\section{RESULTS}

\section{Immunohistochemical Analysis of NLRP3 Inflammasomes}

In the present study, the localization of the core protein NLRP3 and the adaptor protein ASC of inflammasomes were examined through immunohistochemical staining (Figures 1, 2), and the relative expressions were present in Tables $\mathbf{1}, \mathbf{2}$. The results showed NLRP3 mainly expressed in the outside of intrafollicular fluid in the ovaries with PMSG-52 h treatment (Figure 1), which was similar with the pattern of ASC expressions (Figure 2).

\section{Expression Changes of NLRP3 Inflammasomes in the Ovary During the Follicular Development Induced by PMSG}

For confirming the above findings, the expressions of NLRP3 and ASC were further examined by Western blotting (Figure 3) and the results also suggested NLRP3 and ASC mainly expressed in the ovaries after PMSG-52 $\mathrm{h}$ treatment, indicating the activation of NLRP3 inflammasomes this time. Therefore, the expressions of cleaved-caspase-1 were detected (Figure 4) and then found a significant decrease of pro-caspase-1 expression and a dramatic increase of cleaved-caspase- 1 expression in the ovaries after PMSG-52 $\mathrm{h}$ treatment (Figure 4), implying NLRP3 inflammasomes may be involved in the following ovulation induced by MPSG.

\section{Expression and Localization of IL-1 $\beta$ in the Ovary During the Follicular Development Induced by PMSG}

Given IL-1 $\beta$ production resulted from the activation of NLRP3 inflammasomes, the present study examined the expression (Figure 5 and Table 3) and localization (Figure 5) of IL-1 $\beta$ in the ovary during the follicular development induced by PMSG and the results further demonstrated IL-1 $\beta$ mainly expressed in the outside of intrafollicular fluid (Figure 5) and significantly increased (Figure 6) in the ovaries with PMSG-52 h treatment, which were similar with the expression pattern of NLRP3 and ASC proteins.

\section{Activity Changes of Caspase-1 in the Ovary During the Follicular Development Induced by PMSG}

Furthermore, the present study also examined caspase-1 activity (Figure 7A) and IL-1b production (Figure 7B) through ELISA kits and further found a significant increase of
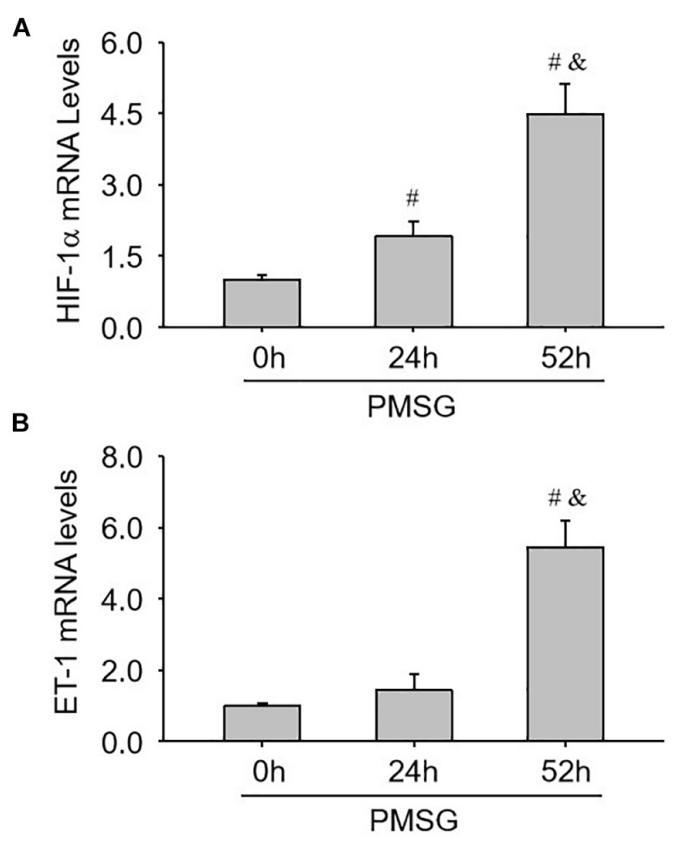

FIGURE 8 | Expression changes of HIF- $1 \alpha$ and ET-1 mRNA in the ovaries during the follicular development induced by PMSG. (A) The relative mRNA levels of HIF- $1 \alpha$ by real-time PCR analysis. (B) The relative mRNA levels of ET-1 by real-time PCR analysis. Each value represents the mean $\pm \mathrm{SE}$. One-way analysis of variance (ANOVA) was used to analyze the data, followed by a Tukey's multiple range test. $n=6$. ${ }^{\#} P<0.05$, vs. PMSH-0 h; ${ }^{\&} P<0.05$, vs. PMSH-24 h. 
caspase-1 activity (Figure 7A) and a dramatic increase of IL-1 $\beta$ production (Figure 7B), suggesting NLRP3 inflammasomes were activated and involved in the following ovulation induced by MPSG.

\section{Expression Changes of Ovulation-Related Genes in the Ovary During the Follicular Development Induced by PMSG}

Finally, the expressions of ovulation-related genes, HIF- $1 \alpha$ (Figure 8A) and ET-1 (Figure 8B), were examined through real time PCR and found their expressions also increased significantly in the ovaries with PMSG-52 h treatment (Figure 8), suggesting the activation of NLRP3 inflammasomes may take participate in the ovulatory process with the detailed mechanism to be further clarified. Together, the present study not only summarized the expression changes of NLRP3 inflammasomes during the follicular development (Figure 9A), but also put forward the possible role of NLRP3 inflammasomes during the following ovulation (Figure 9B).

\section{DISCUSSION}

The present study mainly examined that expression of NLRP3 inflammasomes during the follicular development induced by PMSG, clearly demonstrating that the activation of NLRP3 inflammasomes may take participate in the following process of ovulation, which will help to further clarify the ovulatory mechanism in mammals.

Pregnant mare serum gonadotropin is mainly similar to FSH and widely used for superovulation (Ma et al., 1997; Tarín et al., 2002; Nie et al., 2018; Kim et al., 2019), the present study therefore, utilized PMSG to trigger the follicular development and the following ovulation for examining the expression changed of NLRP3 inflammasomes during the follicular development and clarifying the possible role of NLRP3 inflammasomes during the following ovulation. It's well-known that inflammation is involved in the ovulatory process (Gonzalez et al., 2018; Duffy et al., 2019; Poulsen et al., 2019; Snider and Wood, 2019), but the detailed mechanism regulating the crosstalk between the inflammatory and ovulatory processes still remains unclear. Therefore, the contribution of the inflammatory processes to the ovulation in mammalian ovaries recently attracted more and more attention (Gonzalez et al., 2018; Duffy et al., 2019; Poulsen et al., 2019; Snider and Wood, 2019). Duffy et al. (2019) Found the parallel of the ovulatory and inflammatory processes with many common features, ovarian granulosa cells executed an inflammatory reaction during the ovulation (Poulsen et al., 2019), obesity reduced ovarian oocyte quality with inflammation (Snider and Wood, 2019), inflammatory markers in the follicular fluid were correlated with body mass index (Gonzalez et al., 2018), and so on. Given the important role of NLRP3 inflammasomes in the inflammatory responses (Zhang et al., 2015; Zhu et al., 2016), the present study examined expressions of NLRP3 inflammasomes during the follicular development induced by PMSG and found NLRP3 and ASC
A

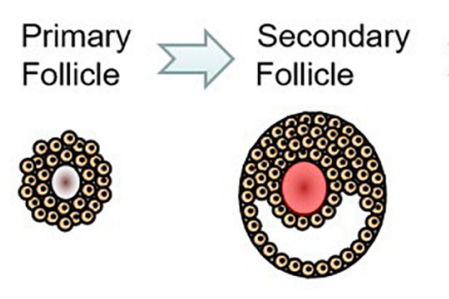

- Granulosa Cell $\bigcirc$ Oocyte

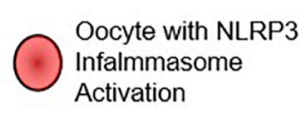

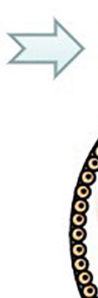

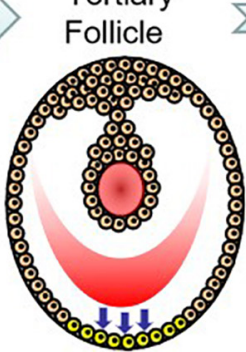

NLRP3 Inflammasome

Expression in

Follicular Liquid
Follicle Ruptured

Oocyte Released

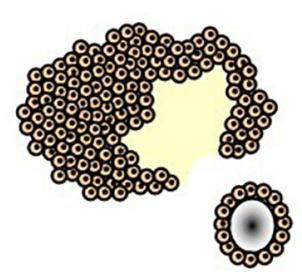

Effect Direction

$\Rightarrow$ of NLRP3 Inflammasome
Target cells of

8 NLRP3

Inflammasome

B

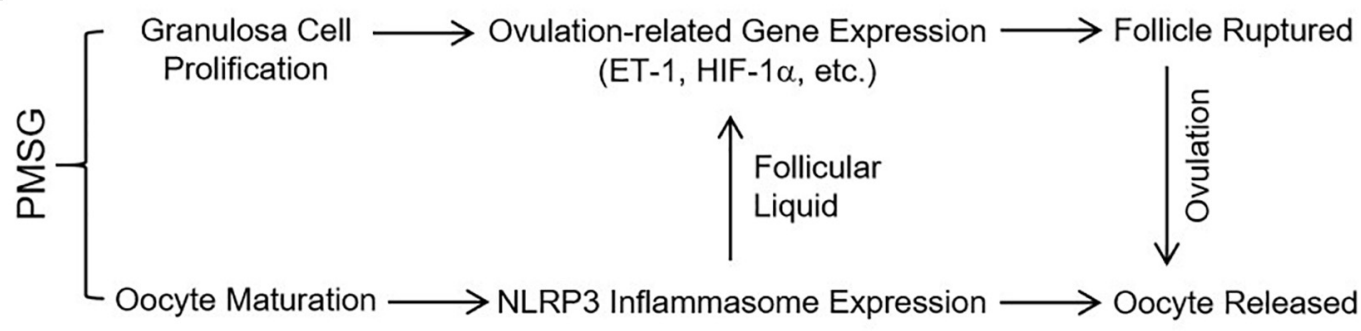

FIGURE 9 | Expression pattern and possible role of NLRP3 inflammasomes in the ovaries during the follicular development induced by PMSG. (A) Expression pattern of NLRP3 inflammasomes in the ovarian follicles during the follicular development induced by PMSG. (B) Possible role of NLRP3 inflammasomes in the PMSG-treated ovaries during the follicular development and the following ovulation. 
expressions significantly increased after PMSG-52 h treatment, which were consistent with the dramatic increases of caspase- 1 activity and IL- $1 \beta$ production, suggesting NLRP3 inflammasomes were activated before the ovulation and involved in the following ovulation. This findings of NLRP3 inflammasome activation could be a new mechanism regulating the ovulatory process in mammals.

Notably, the present study also found that expression and activation of NLRP3 inflammasomes in ovarian oocytes were prior to those in the follicular fluid, suggesting which was regulated by oocyte quality or maturation. Recent investigations have also demonstrated the coordination of ovulation with oocyte maturation and the decisive effects of oocytes during the ovulatory process (Robker et al., 2018). Interestingly, the present results showed that expressions and activation of NLRP3 inflammasomes in the outside of intrafollicular fluid after PMSG-52 h treatment, suggesting the regional responses in the ovulatory follicles (Duffy et al., 2019), which contributed to the apical stretching and thinning (Duffy et al., 2019). Previous studies have indicated ETs facilitated follicular rupture at the apex (Ko et al., 2006; Choi et al., 2011; Cacioppo et al., 2017) and HIF-1 $\alpha$ regulated ovulation-related gene expression (Zhang Z. et al., 2011; Wang et al., 2012; Wang et al., 2015) during the ovulatory process. Therefore, the present study further examined the expressions of HIF-1 $\alpha$ and ET-1 and found their expression patterns were similar with NLRP inflammasomes, demonstrating the possible contribution of NLRP inflammasomes to the ovulation.

Furthermore, the correlation between non-steroidal antiinflammatory drug use and reversible infertility in women indicated the ovulatory processes with inflammatory responses (Akil et al., 1996; Mendonça et al., 2000; Duffy et al., 2019), further implying the activation of NLRP3 inflammasomes may play an important role in the regulation of ovarian ovulation in mammals. Although the present findings is very interesting and helpful for further understanding the molecular regulation of the ovulatory process, some more detailed investigations need to be performed in the near future for finally clarifying the relationship between the activation of NLRP3 inflammasomes and the ovulation of mammalian ovaries.

In summarize, the present study firstly demonstrated the expression and activation of NLRP3 inflammasomes during the follicular development induced by PMSG, which may contribute to the following ovulation. Furthermore, enhanced

\section{REFERENCES}

Abais, J. M., Xia, M., Zhang, Y., Boini, K. M., and Li, P. L. (2015). Redox regulation of NLRP3 inflammasomes: ROS as trigger or effector? Antioxid. Redox. Signal. 22, 1111-1129. doi: 10.1089/ars.2014.5994

Ahechu, P., Zozaya, G., Martí, P., Hernández-Lizoáin, J. L., Baixauli, J., Unamuno, X., et al. (2018). NLRP3 Inflammasome: a possible link between obesityassociated low-grade chronic inflammation and colorectal cancer development. Front. Immunol. 9:2918. doi: 10.3389/fimmu.2018.02918

Akil, M., Amos, R. S., and Stewart, P. (1996). Infertility may sometimes be associated with NSAID consumption. Br. J. Rheumatol. 35, 76-78.

Boini, K. M., Xia, M., Abais, J. M., Li, G., Pitzer, A. L., Gehr, T. W., et al. (2014). Activation of inflammasomes in podocyte injury of mice on the high fat understanding of the contribution and regulation of NLRP3 inflammasomes during ovarian ovulation will be helpful to treat anovulatory infertility, like luteinized unruptured follicle syndrome and polycystic ovary syndrome.

\section{DATA AVAILABILITY STATEMENT}

The datasets generated for this study are available on request to the corresponding author.

\section{ETHICS STATEMENT}

The experimental protocol was approved in accordance with the Guide for the Care and Use of Laboratory Animals prepared by the Institutional Animal Care and Use Committee, Fujian Normal University (IACUC-20180011).

\section{AUTHOR CONTRIBUTIONS}

$\mathrm{ZZ}$ and FW designed the study. FW and YZ wrote the manuscript. ZZ revised the manuscript. All authors performed the experiments, analyzed the data, interpreted the data, discussed the results, read, and approved the final version of the manuscript for publication.

\section{FUNDING}

This study was supported by the Fujian Provincial Natural Science Foundation (2017J01626, 2018J01721, and 2018J01722), Fujian Province Science and Technology Project of The Education Department (JZ160426), National Training Program of Innovation and Entrepreneurship for Undergraduates (201910394023), and the Educational Reform Project of Fujian Normal University (Y201809 and P201801016).

\section{ACKNOWLEDGMENTS}

The authors thank the staff in the Laboratory Animal Center of Fujian Normal University for giving us kind help during the present experiment.

diet: effects of ASC gene deletion and silencing. Biochim. Biophys. Acta. 1843, 836-845. doi: 10.1016/j.bbamcr.2014.01.033

Cacioppo, J. A., Lin, P. P., Hannon, P. R., McDougle, D. R., Gal, A., and Ko, C. (2017). Granulosa cell endothelin-2 expression is fundamental for ovulatory follicle rupture. Sci. Rep. 7:817. doi: 10.1038/s41598-017-00943-w

Chen, Y., Pitzer, A. L., Li, X., Li, P. L., Wang, L., and Zhang, Y. (2015). Instigation of endothelial Nlrp3 inflammasome by adipokine visfatin promotes inter-endothelial junction disruption: role of HMGB1. J. Cell. Mol. Med. 19, 2715-2727. doi: 10.1111/jcmm.12657

Chen, Y., Wang, L., Pitzer, A. L., Li, X., Li, P. L., and Zhang, Y. (2016). Contribution of redox-dependent activation of endothelial Nlrp3 inflammasomes to hyperglycemia-induced endothelial dysfunction. J. Mol. Med. 94, 1335-1347. doi: 10.1007/s00109-016-1481-5 
Choi, D. H., Kim, E. K., Kim, K. H., Lee, K. A., Kang, D. W., Kim, H. Y., et al. (2011). Expression pattern of endothelin system components and localization of smooth muscle cells in the human pre-ovulatory follicle. Hum. Reprod. 26, 1171-1180. doi: 10.1093/humrep/der066

de Alba, E. (2019). Structure, interactions and self-assembly of ASC-dependent inflammasomes. Arch. Biochem. Biophys. 670, 15-31. doi: 10.1016/j.abb.2019. 05.023

Duffy, D. M., Ko, C., Jo, M., Brannstrom, M., and Curry, T. E. (2019). Ovulation: parallels with inflammatory processes. Endocr. Rev. 40, 369-416. doi: 10.1210/ er.2018-2075

Espey, L. L. (1980). Ovulation as an inflammatory reaction-a hypothesis. Biol. Reprod. 22, 73-106. doi: 10.1095/biolreprod22.1.73

Gonzalez, M. B., Lane, M., Knight, E. J., and Robker, R. L. (2018). Inflammatory markers in human follicular fluid correlate with lipid levels and body mass index. J. Reprod. Immunol. 130, 25-29. doi: 10.1016/j.jri.2018.08.005

Groslambert, M., and Py, B. F. (2018). Spotlight on the NLRP3 inflammasome pathway. J. Inflamm. Res. 11, 359-374. doi: 10.2147/JIR.S141220

Jiang, J., Qi, L., Wei, Q., and Shi, F. (2018). Effects of daily exposure to saccharin sodium and rebaudioside a on the ovarian cycle and steroidogenesis in rats. Reprod. Toxicol. 76, 35-45. doi: 10.1016/j.reprotox.2017.12.006

Kim, H., Moon, C., Ahn, M., Lee, Y., Kim, H., Kim, S., et al. (2005). Expression of nitric oxide synthase isoforms in the porcine ovary during follicular development. J. Vet. Sci. 6, 97-101.

Kim, J., Sun, S., Lee, D., Youk, H., and Yang, H. (2019). Gonadotropin regulates NUCB2/nesfatin-1 expression in the mouse ovary and uterus. Biochem. Biophys. Res. Commun. 513, 602-607. doi: 10.1016/j.bbrc.2019.04.008

Ko, C., Gieske, M. C., Al-Alem, L., Hahn, Y., Su, W., Gong, M. C., et al. (2006). Endothelin-2 in ovarian follicle rupture. Endocrinology 147, 1770-1779. doi: 10.1210/en.2005-1228

Komada, T., and Muruve, D. A. (2019). The role of inflammasomes in kidney disease. Nat. Rev. Nephrol. 15, 501-520. doi: 10.1038/s41581-019-0158-z

Komatsu, K., and Masubuchi, S. (2016). Observation of the dynamics of follicular development in the ovary. Reprod. Med. Biol. 16, 21-27. doi: 10.1002/rmb2. 12010

Li, X., Zhang, Y., Xia, M., Gulbins, E., Boini, K. M., and Li, P. L. (2014). Activation of Nlrp3 inflammasomes enhances macrophage lipid-deposition and migration: implication of a novel role of inflammasome in atherogenesis. PLoS One 9:e87552. doi: 10.1371/journal.pone.0087552

Ma, S., Kalousek, D. K., Yuen, B. H., and Moon, Y. S. (1997). Investigation of effects of pregnant mare serum gonadotropin (PMSG) on the chromosomal complement of CD-1 mouse embryos. J. Assist. Reprod. Genet. 14, 162-169. doi: $10.1007 / \mathrm{bf} 02766134$

Mariathasan, S., Newton, K., Monack, D. M., Vucic, D., French, D. M., Lee, W. P., et al. (2004). Differential activation of the inflammasome by caspase-1 adaptors ASC and Ipaf. Nature 430, 213-218. doi: 10.1038/nature02664

Mendonça, L. L., Khamashta, M. A., Nelson-Piercy, C., Hunt, B. J., and Hughes, G. R. (2000). Non-steroidal anti-inflammatory drugs as a possible cause for reversible infertility. Rheumatology 39, 880-882. doi: 10.1093/rheumatology/39. 8.880

Nie, X., Dai, Y., Zheng, Y., Bao, D., Chen, Q., Yin, Y., et al. (2018). Establishment of a mouse model of premature ovarian failure using consecutive superovulation. Cell. Physiol. Biochem. 51, 2341-2358. doi: 10.1159/000495895

Poulsen, L. C., Englund, A. L. M., Wissing, M. L. M., Yding Andersen, C., Borup, R., and Grøndahl, M. L. (2019). Human granulosa cells function as innate immune cells executing an inflammatory reaction during ovulation: a microarray analysis. Mol. Cell. Endocrinol. 486, 34-46. doi: 10.1016/j.mce.2019.02.014

Qi, L., Jiang, J., Jin, P., Kuang, M., Wei, Q., Shi, F., et al. (2018). Expression patterns of claudin-5 and its related signals during luteal regression in pseudopregnant rats: the enhanced effect of additional PGF treatment. Acta. Histochem. 120, 221-227. doi: 10.1016/j.acthis.2018.02.001

Robker, R. L., Hennebold, J. D., and Russellm, D. L. (2018). Coordination of ovulation and oocyte maturation: a good egg at the right time. Endocrinology 159, 3209-3218. doi: 10.1210/en.2018-2485

Shi, F., Stewart, R. L. Jr., Perez, E., Chen, J. Y., and LaPolt, P. S. (2004). Cellspecific expression and regulation of soluble guanylyl cyclase alpha 1 and beta 1 subunits in the rat ovary. Biol. Reprod. 70, 1552-1561. doi: 10.1095/biolreprod. 103.025510

Snider, A. P., and Wood, J. R. (2019). Obesity induces ovarian inflammation and reduces oocyte quality. Reproduction doi: 10.1530/REP-18-0583 [Epub ahead of print].

Takahashi, M. (2019). Role of NLRP3 inflammasome in cardiac inflammation and remodeling after myocardial infarction. Biol. Pharm. Bull. 42, 518-523. doi: 10.1248/bpb.b18-00369

Tarín, J. J., Pérez-Albalá, S., and Cano, A. (2002). Stage of the estrous cycle at the time of pregnant mare's serum gonadotropin injection affects the quality of ovulated oocytes in the mouse. Mol. Reprod. Dev. 61, 398-405. doi: 10.1002/ mrd. 10042

Wang, F., Zhang, Z., Wang, Z., Xiao, K., Wang, Q., Su, J., et al. (2015). Expression and clinical significance of the HIF-1 $\alpha /$ ET-2 signaling pathway during the development and treatment of polycystic ovary syndrome. J. Mol. Histol. 46, 173-181. doi: 10.1007/s10735-015-9609-9604

Wang, L., Chen, Y., Li, X., Zhang, Y., Gulbins, E., and Zhang, Y. (2016). Enhancement of endothelial permeability by free fatty acid through lysosomal cathepsin B-mediated Nlrp3 inflammasome activation. Oncotarget 7, 7322973241. doi: 10.18632/oncotarget.12302

Wang, Z., Zhang, Z., Wu, Y., Chen, L., Luo, Q., Zhang, J., et al. (2012). Effects of echinomycin on endothelin-2 expression and ovulation in immature rats primed with gonadotropins. Exp. Mol. Med. 44, 615-621. doi: 10.3858/emm. 2012.44.10.070

Wei, Q., Fedail, J. S., Kong, L., Zheng, K., Meng, C., Fadlalla, M. B., et al. (2018). Thyroid hormones alter estrous cyclicity and antioxidative status in the ovaries of rats. Anim. Sci. J. 89, 513-526. doi: 10.1111/asj.12950

Yang, Q., Liu, R., Yu, Q., Bi, Y., and Liu, G. (2019). Metabolic regulation of inflammasomes in inflammation. Immunology 157, 95-109. doi: 10.1111/imm. 13056

Zhang, C., Boini, K. M., Xia, M., Abais, J. M., Li, X., Liu, Q., et al. (2012). Activation of nod-like receptor protein 3 inflammasomes turns on podocyte injury and glomerular sclerosis in hyperhomocysteinemia. Hypertension $\quad 60, \quad 154-162$. doi: 10.1161/HYPERTENSIONAHA.111. 189688

Zhang, W., Wei, Q. W., Wang, Z. C., Ding, W., Wang, W., and Shi, F. X. (2011). Cell-specific expression and immunolocalization of nitric oxide synthase isoforms and the related nitric oxide/cyclic GMP signaling pathway in the ovaries of neonatal and immature rats. J. Zhejiang Univ. Sci. B 12, 55-64. doi: 10.1631 /jzus.B1000174

Zhang, Y., Li, X., Pitzer, A. L., Chen, Y., Wang, L., and Li, P. L. (2015). Coronary endothelial dysfunction induced by nucleotide oligomerization domain-like receptor protein with pyrin domain containing 3 inflammasome activation during hypercholesterolemia: beyond inflammation. Antioxid. Redox. Signal. 22, 1084-1096. doi: 10.1089/ars.2014.5978

Zhang, Z., Yin, D., and Wang, Z. (2011). Contribution of hypoxia-inducible factor1a to transcriptional regulation of vascular endothelial growth factor in bovine developing luteal cells. Anim. Sci. J. 82, 244-250. doi: 10.1111/j.1740-0929.2010. 00832.x

Zhu, Q., Li, X. X., Wang, W., Hu, J., Li, P. L., Conley, S., et al. (2016). Mesenchymal stem cell transplantation inhibited high salt-induced activation of the NLRP3 inflammasome in the renal medulla in Dahl S rats. Am. J. Physiol. Renal. Physiol. 310, F621-F627. doi: 10.1152/ajprenal.00344. 2015

Conflict of Interest: The authors declare that the research was conducted in the absence of any commercial or financial relationships that could be construed as a potential conflict of interest.

Copyright $\odot 2019$ Zhang, Wang and Zhang. This is an open-access article distributed under the terms of the Creative Commons Attribution License (CC BY). The use, distribution or reproduction in other forums is permitted, provided the original author(s) and the copyright owner(s) are credited and that the original publication in this journal is cited, in accordance with accepted academic practice. No use, distribution or reproduction is permitted which does not comply with these terms. 